Javier Livas:

\title{
Trust, Technology and Requisite Variety
}

\section{Abstract:}

The Cybernetic State is about using what we know now about cybernetics to create a better society for all. It is about satisfying people's needs from a platform of individual freedom and responsibility. It is also about using computers wisely. In order to do that, technology has to produce transparency so that as many people as possible contribute to the new social order. To create a transparent government implies making it easy to understand and very dependable.

\section{Author:}

Javier A. Livas Cantu

- Javier Cantu has worked as banker, attorney and consultant. He has been heavily involved in political activism for freedom and democracy.

- Javier may be contacted at javierlivas@mac.com 
My good friend and mentor, Stafford Beer once gave a lecture where he explained what The Cybersyn Project had been in Chile in the early 1970s and how it was going to work. As time has gone by, the technology for creating a government with control in real time has improved by leaps and bounds. It would be much easier to do it now, unfortunately what has not evolved at the same pace is the cybernetic knowledge of those in power.

The key to understanding governance is knowing the Law of Requisite Variety or Ashby's Law. It simply says that in matters of control: "Only variety absorbs variety." Though this may sound tautological or self-evident, what it really means is that in order to control the perturbation of some system, its variety (or different states it can adopt), must be matched by the variety of the control system. A thermostat shows as much "variety" as the climate it is trying to control (up to a certain point of course).

I have another explanation that I call the variety equation. Everything that you want to do or transform requires a given amount of information, or control, or variety. This resulting quantity has to be matched by the control system or regulator.

In the design of the State individual freedoms are given the outmost priority. In fact, the Constitution is the set of limitations (controls) that the people impose on the Government in order to protect their freedoms. In theory at least, there is a balance between our freedom and our obligations to the State. If all works well then the variety equation is satisfied.

However there are many variety strategies that can lead to the same result. In that case you would prefer the solution that maximizes freedom and still gets the final result the people want.

For instance, let's assume that the government wants to stop criminal activity. It has two strategies. Hire many policemen or educate the young to be law-abiding citizens all the time.

Golf relies on each golfer to apply penalties on himself. This is the extreme of what an ideal society would want. This is ethics and trust at its maximum expression. The other extreme is have a policeman for every citizen and then police watching other police, and so on.

The police do not come alone. They bring with them attorneys, judges, prosecutors, jails, and so on. All these inputs try to achieve requisite variety, which means being able to dissuade people not to commit crimes.

The other strategy is to educate people to always act in an ethical manner. If everybody acted correctly you would not need the judiciary and everything that comes with it. By ethical behavior I mean doing the right thing right and at the right time. Just as a golfer is expected to do. Professionals golfers do it all the time!

What happens in real life is that every society is trying hard to teach children to learn to behave correctly. But since this is hard to achieve, then much noise is produced and the variety of situation explodes beyond any form of control.

Technology is a control amplifier. Trust is also on the same side of the control equation, opposite the perturbation side. Together, trust and technology they can prevent crime from happening by remembering who the bad guys are. The problem is on the opposite side with its perturbation variety demanding to be matched or else. You can fix certain problems by throwing money at them, but sometimes it just makes the problem worse.

Ideally any country should try to create a culture where the government produces wise laws and these are respected and obeyed by everybody. It is to a great extent a problem of good communication. Law and the judiciary must act as a last resort, after education and negotiation has run its course without success. 